Meta

Journal des traducteurs

Translators' Journal

\title{
El papel de la traducción en The Arte of English Poesie (1589)
}

\section{Rocío G. Sumillera}

Volume 56, numéro 4, décembre 2011

URI : https://id.erudit.org/iderudit/1011255ar

DOI : https://doi.org/10.7202/1011255ar

Aller au sommaire du numéro

\section{Éditeur(s)}

Les Presses de l’Université de Montréal

ISSN

0026-0452 (imprimé)

1492-1421 (numérique)

Découvrir la revue

Citer cet article

Sumillera, R. G. (2011). El papel de la traducción en The Arte of English Poesie (1589). Meta, 56(4), 833-851. https://doi.org/10.7202/1011255ar

\section{Résumé de l'article}

Le présent article propose une analyse approfondie du rôle central joué par la traduction dans The Arte of English Poesie (1589), de George Puttenham. Un certain nombre de traductions et de pensées sur la traduction se trouvant dans cet ouvrage ont été analysées et contextualisées. Une attention particulière est portée à la traduction en anglais des nombreux vers latins inclus dans le volume, ainsi qu'aux noms de plus d'une centaine de figures rhétoriques qui y sont expliquées. Il sera ainsi possible de montrer que la traduction remplit une fonction essentielle dans le processus d'adaptation du contenu rhétorique de The Arte of English Poesie au profil des lecteurs cibles, c'est-à-dire des courtisans oisifs qui ne connaissaient pas les langues classiques mais qui étaient épris de lecture ou d'écriture de poésie. 


\title{
El papel de la traducción en The Arte of English Poesie (1589)
}

\author{
ROCÍO G. SUMILLERA \\ Universidad de Granada, Granada, España \\ sumille@correo.ugr.es
}

\begin{abstract}
RÉSUMÉ
Le présent article propose une analyse approfondie du rôle central joué par la traduction dans The Arte of English Poesie (1589), de George Puttenham. Un certain nombre de traductions et de pensées sur la traduction se trouvant dans cet ouvrage ont été analysées et contextualisées. Une attention particulière est portée à la traduction en anglais des nombreux vers latins inclus dans le volume, ainsi qu'aux noms de plus d'une centaine de figures rhétoriques qui y sont expliquées. Il sera ainsi possible de montrer que la traduction remplit une fonction essentielle dans le processus d'adaptation du contenu rhétorique de The Arte of English Poesie au profil des lecteurs cibles, c'est-à-dire des courtisans oisifs qui ne connaissaient pas les langues classiques mais qui étaient épris de lecture ou d'écriture de poésie.
\end{abstract}

\begin{abstract}
This article thoroughly analyzes the central role of translation in George Puttenham's The Arte of English Poesie (1589), studying and contextualizing the different instances of translation and reflections on translating that are present in the book. By paying special attention to the translation into English of the numerous Latin lines included in the volume, as well as to the names of over a hundred rhetorical figures explained in it, it will be demonstrated that in The Arte of English Poesie translation has an essential function within the process of adjusting the work's rhetorical teachings to the profile of its target readership: idle courtiers ignorant of classical languages but highly interested in reading or composing poetry.
\end{abstract}

\section{MOTS-CLÉS/KEYWORDS}

Renaissance anglaise, figures rhétoriques, langues vernaculaires, poésie English Renaissance, rhetorical figures, vernacular languages, poetry

\section{Introducción}

En su artículo Pour une historiographie des théories de la traduction: questions de méthode, D’hulst (1995) subrayaba la necesidad de que, con el fin de lograr una visión más completa de la evolución del concepto de traducción a lo largo del tiempo, la historia de la traducción se hiciera eco de reflexiones sobre la traducción misma procedentes de disciplinas vecinas como la poética, la gramática, o la retórica. La idea subyacente es que las reflexiones sobre la traducción y el papel del traductor no han estado exclusivamente localizadas en prefacios o dedicatorias a traducciones, sino que más bien se han encontrado dispersas en obras de variada naturaleza, de ahí la necesidad de salir del campo estrictamente de la traducción para encontrar opiniones y consideraciones sobre la misma. Así, por ejemplo, las conexiones entre las disciplinas de la traducción y la retórica han sido efectivamente estudiadas, normalmente 
prestando especial atención al tema de la didáctica, pues incluso durante el Renacimiento seguía en pie la creencia heredada de la Antigüedad clásica de que el conocimiento de la retórica era de gran utilidad para los traductores, y que los ejercicios de traducción complementaban la formación de los rétores (Steiner 1975: 7; Bassnett-McGuire 1980: 51-52; Rener 1989: 182-260; Oliver 1995: 23-26; Arduini 2000: 159-178; Chico Rico 2002). A pesar de los estudios llevados a cabo en esta dirección, aún quedan por revisar numerosas retóricas que encierran nociones teóricas sobre la traducción muy jugosas para los interesados en la relación entre ambas disciplinas. Precisamente a este grupo pertenece The Arte of English Poesie (1589), obra de poética/retórica escrita por el inglés George Puttenham (1529-1590/1591) ${ }^{1}$.

No es sorprendente que nociones teóricas sobre la traducción se reflejen en una obra de poética/retórica publicada en la Inglaterra del siglo XVI. Al fin y al cabo, este es un momento de frenética actividad traductora, tanto de otras lenguas vernáculas al inglés, como de las lenguas clásicas ${ }^{2}$. Paralelamente, la retórica se convierte en el centro de atención de los estudiosos y ocupa un lugar privilegiado en escuelas y universidades. Si Vickers habla de una fragmentación de la retórica durante la Edad Media, no puede sino referirse al Renacimiento como el momento de su «reintegración» (Vickers 1990: 254); no en vano, fue entonces cuando "Occidente accedió a todo el corpus de la literatura retórica griega a través de los textos originales o de las traducciones latinas y vernáculas» (Kristeller 1999: 14). En estas circunstancias, la figura del traductor y del rétor confluyen en diversas ocasiones, pues los rétores poseían el conocimiento necesario de las lenguas clásicas para emprender traducciones de originales escritos en ellas. Así, Sir Thomas Elyot (1490-1546) tradujo obras de Isócrates o Plutarco, al igual que Thomas Wilson (c.1525-1581) lo hizo de otras firmadas por Aristóteles, Demóstenes, Cicerón o Quintiliano.

George Puttenham, en cambio, no es reconocido como traductor, pues a parte de The Arte, las únicas obras suyas que han llegado hasta nuestros días han sido Partheniades, compilación de diecisiete poemas en distintos tipos de versos dedicados a Isabel I como regalo de año nuevo ${ }^{3}$, y A Justificacion of Queene Elizabeth in relacion to the affaire of Mary queene of Scottes, texto en prosa que defiende la decisión del gobierno de Isabel I de condenar a muerte a María Estuardo (María I de Escocia). Esto no quiere decir, sin embargo, que Puttenham no tradujera ni que no reflexionara acerca de la labor traductora, ya que The Arte está salpicada de momentos en los que hace gala de sus habilidades como traductor y de sus reflexiones acerca de dicha actividad.

Partiendo de estos presupuestos, el presente artículo analiza sistemáticamente los momentos en que la traducción irrumpe en The Arte, ya sea en forma de reflexiones teóricas, o de traducciones al inglés llevadas a cabo por Puttenham. Asimismo, contextualiza el concepto de traducción manejado por el autor inglés al relacionarlo con el utilizado por algunos de los traductores del XVI, al tiempo que lo coteja con las características de sus propias traducciones con el fin de confirmar si su práctica de la traducción es coherente con sus nociones teóricas o si se produce un desencuentro entre ambas. Por otra parte, este escrito evalúa la importancia que adquiere la traducción en una obra como The Arte, una poética/retórica en lengua vernácula dirigida a unos lectores cortesanos no versados en las lenguas clásicas pero con ciertas inquietudes poéticas. Teniendo esto en mente, se verá cómo la traducción se reviste en la obra de Puttenham de una finalidad pedagógica, contribuyendo de 
manera esencial a lograr la necesaria adecuación entre el contenido del libro y los lectores a los que iba dirigido, quienes, en palabras de Puttenham, no son eruditos ni estudiantes de retórica con pretensiones de dedicarse a la literatura profesionalmente, sino «Ladies and young Gentlewomen, or idle Courtiers, desirous to become skilful in their owne mother tongue» (Puttenham 1589/1970: 132).

\section{El concepto de traducción en The Arte of English Poesie}

The Arte of English Poesie, que versa sobre el arte de la poesía prestando especial atención a la creación poética en lengua inglesa, está dividido en tres libros: «Of Poets and Poesie», «Of Proportion Poetical», y «Of Ornament». «Of Poets and Poesie» incluye consideraciones generales acerca de la poesía y los poetas, una defensa del arte de la poesía, una breve historia de la evolución de la poesía y de la manera en que han sido vistos los poetas, y una valoración de los mejores poetas ingleses. El segundo libro, "Of Proportion Poetical», discute asuntos como la proporción, lo adecuado en poesía en términos de extensión, tono, tema, ritmo, rima o estrofa, comenta cómo adaptar los metros clásicos a la poesía escrita en inglés, y compara las características del verso griego y latino con las del inglés. Finalmente, el tercer libro, «Of Ornament», considera el lenguaje y estilo que debe usar el poeta, y, por supuesto, el ornamento poético: las figuras retóricas ${ }^{4}$. No en vano, críticos como Peter Mack han considerado a este último libro de The Arte un manual de estilo (Mack 2002: 77).

A pesar de ser un tratado de poética/retórica, la traducción es un elemento omnipresente en The Arte. Tanto es así que, ya en la primera página, George Puttenham expone sus ideas acerca de la relación entre traducción y poesía, y de la labor del traductor de poesía en comparación con la del poeta. El argumento básico del que Puttenham parte es que el poeta es un maker, un creador: «A Poet is as much as to say as a maker» (Puttenham 1589/1970: 3) $)^{5}$. El hecho de que el poeta sea un creador implica un esfuerzo para su imaginación que no exige la labor del traductor de poesía, quien es visto por Puttenham como un mero versificador:

Even so the very Poet makes and contrives out of his owne braine, both the verse and matter of his poeme, and not by any foreine copie or example, as doth the translator, who therefore may well be sayd a versifier, but not a Poet. (Puttenham 1589/1970: 3)

En otras palabras, el elemento que diferencia a la traducción de la poesía es que la primera trabaja con un modelo al que tiene que seguir y copiar en otra lengua, mientras que la segunda es un ejercicio de las facultades creativas del poeta; el traductor copia, mientras que el poeta crea. De esta manera, la labor del traductor queda reducida a la mera versificación: a la preocupación por encajar un contenido que ya le es dado en una estructura definida utilizando una lengua diferente. Visto desde esta perspectiva, el trabajo del versificador parece ser más propio de la matemática que del arte, y estar más cercano a un trabajo automático que a una actividad que requiere de las facultades creativas de quien la lleva a cabo. Puttenham coloca, pues, la labor del poeta sobre la del traductor.

La aparentemente sencilla definición de poeta que Puttenham propone en las tres líneas de la cita anterior, se ve, sin embargo, matizada y problematizada en las frases que la siguen, en las que también se reconoce al poeta como imitador: 
And neverthelesse without any repugnancie at all, a Poet may in some sort be said a follower or imitator, because he can expresse the true and lively of every thing is set before him, and which he taketh in hand to describe: and so in that respect is both a maker and a counterfaitor: and Poesie an art not only of making, but also of imitation. (Puttenham 1589/1970: 3)

Debido a que Puttenham define la labor del traductor de poesía a partir de la del poeta, en el momento en que matiza su concepción de la poesía, la de la traducción parece verse afectada también. Si en un principio la creatividad era lo más característico de la poesía, y la copia o la imitación, el terreno propio de la traducción, cuando Puttenham afirma que la poesía puede salir del área de la creación y pasar al de la imitación, surge la pregunta de por qué no va a poder también la traducción cruzar la frontera de la imitación y participar de la creatividad humana.

El hecho de que Puttenham reconozca al poeta como imitador indica que el autor inglés se hace eco de las teorías propugnadas por la filosofía neoplatónica, la cual ejerció una gran influencia sobre el pensamiento medieval y renacentista (Howatson y Chilvers 1993). El neoplatonismo, que pretendió aunar la filosofía platónica con el aristotelismo así como con otras corrientes de pensamiento de la Antigüedad clásica, llevó a cabo una reinterpretación positiva del concepto platónico de poesía como imitación o mímesis, lo cual ya había hecho Aristóteles anteriormente en su Poética pero utilizando argumentos diferentes. Mientras que para Platón la imitación significaba un alejamiento negativo de las Ideas, de la realidad última, los neoplatónicos entienden que la imitación artística, dentro de la que se incluye la imitación poética, transciende del mundo físico al de las apariencias, e imita directamente a la verdad divina (Preminger y Brogan 1993: 912). Es por esta razón por lo que a Puttenham no le avergüenza afirmar («without any repugnancie at all») que la poesía es un arte imitativo ${ }^{6}$.

La concepción de la traducción como imitación dista de ser, por tanto, una idea propia de Puttenham, al estar muy extendida tanto en la Inglaterra como en la Francia del Renacimiento. En Inglaterra, los comentarios sobre traducción más relevantes de la época van unidos a nombres como los de Lawrence Humphrey (c.1527-1590), autor del importante Interpretatio lingvarvm (1559), a George Chapman (c.1559-1634), y, durante la primera mitad del siglo XviI, a Abraham Cowley (c.1618-1667) y Sir John Denham (1615-1669). Sin embargo, en el siglo xVI son traductores franceses como Étienne Dolet (1509-1546), Jacques Peletier du Mans (1517-1582), Thomas Sebillet (1512-1589) y Joachim du Bellay (1522-1560), los que sobresalen en el campo de la teoría, pues en este período los franceses habían reflexionado más acerca de la traducción que los ingleses (Steiner 1975: 13) ${ }^{7}$.

Las consideraciones de los traductores franceses de mediados del siglo XVI constituyen un buen marco de referencia para entender las ideas sobre traducción expuestas por Puttenham. Las opiniones de Jacques Peletier, Thomas Sebillet, o Joachim du Bellay remiten justamente a la concepción de la traducción como imitación. De esta manera, cuando en Art poétique français (1548) Thomas Sebillet aconseja al interesado en emprender una traducción que se fije en aquéllas realizadas por traductores de reconocido prestigio, lo hace en los siguientes términos:

puisque la version n'est rien qu'une imitation, t'y puis-je mieux introduire qu'avec imitation? Imite donc Marot en sa Métamorphose, en son Musée, en ses Psaumes: Salel, en son Iliade: Héroët, en son Androgyne: Des Masures, en son Énéide: Peletier, en son 
Odyssée et Géorgique. Imite tant de divins esprits, qui suivant la trace d'autrui, font le chemin plus doux à suivre, et sont eux-mêmes suivis. (Sebillet en Goyet 1990: 147)

Por su parte, en Art poétique (1555) Peletier califica a la traducción como «la plus vraie espèce d'Imitation» (Peletier en Goyet 1990: 262), y presenta al traductor dependiente de la inventio, la dispositio y la elocutio empleadas por el autor del texto original. Finalmente, Du Bellay, que reserva tres capítulos en Defensa e ilustración de la lengua francesa (1549) para tratar cuestiones de traducción, la concibe como el acto de pasar un texto de una lengua a otra sin posibilidad de transferir de manera intacta la energía o el espíritu del autor del original.

Algunos críticos explican esta posición común a los poetas de La Pléiade diciendo que la disciplina filológica del humanismo les «llevó a descubrir la originalidad innata de cada lengua y su genio intraducible, lo que los lleva a poner de relieve la insuficiencia de cualquier tipo de traducción» (García Peinado 2002: 112). El traductor se compara, por tanto, con un pintor que puede representar el cuerpo de una persona pero no su alma ${ }^{8}$. Otros autores han subrayado que incluso cuando traducción e imitación se asocian en el Renacimiento, la traducción es la que sale perdiendo de las dos, primeramente porque al tomarse la reproducción exacta como fin último de la traducción, dicho fin se muestra siempre inalcanzable; en segundo lugar, porque se percibe que el traductor posee una restringida libertad de movimientos, y, por consiguiente, se piensa que éste se encuentra en una posición de subordinación e incluso de servilismo con respecto al modelo a seguir; y, por último, porque independientemente de su valor, al verse el texto traducido como una copia de una obra original, automáticamente se le coloca en un estatus inferior a ésta (Hermans 1985: 103-104) ${ }^{9}$.

La idea de la traducción como imitación va a su vez de la mano de un escepticismo, por parte de los traductores de La Pléiade, hacia la traducción palabra por palabra. Thomas Sebillet, por ejemplo, da el siguiente consejo al lector/traductor al que dirige el texto: «ne jure tant superstitieusement aux mots de ton auteur, que iceux délaissés pour retenir la sentence, tu ne serves de plus près à la phrase et propriété de ta langue, qu'à la diction de l'étrangère» (Sebillet en Goyet 1990: 146). Du Bellay se dirige también a los traductores con afirmaciones de este tipo: «si vous efforcez exprimer le naif en une autre Langue, observant la loy de traduyre, qui est n'espacier point hors des limites de l'aucteur, vostre diction sera contrainte, froide, \& de mauvaise grace» (Du Bellay 1549/1997: 36). Peletier coincide con sus compatriotas al afirmar lo siguiente:

les Traductions de mot à mot n'ont pas grâce: non qu'elles soient contre la loi de Traduction: mais seulement pour raison que deux langues ne sont jamais uniformes en phrases. Les conceptions sont communes aux entendements de tous hommes: mais les mots et manières de parler sont particuliers aux nations. (Peletier en Goyet 1990: 265)

La traducción palabra por palabra es, además, rechazada por completo por los ingleses George Chapman y, posteriormente, Sir John Denham y Abraham Cowley. Ya en su primera traducción de Homero, Seaven Bookes of the Iliad (1598), publicada nueve años después de The Arte, Chapman afirmaba lo siguiente:

The worth of a skillful and worthy translator is to observe the sentences, figures and formes of speech proposed in his author, his true sence of height, and to adorne them with figures and formes of oration fitted to the originall in the same tongue to which they were translated. (citado en Steiner 1975: 8) 
En cuanto a Denham y Cowley, cuyos comentarios dominan la teoría de la traducción en Inglaterra entre los años 1647 y 1656, han sido considerados unos traductores «ligados a la poesía para quienes el acento va a estar no en la autonomía del original, sino en la estética del poema traducido», lo cual «les obliga a rechazar de plano el principio de la traducción literal» (Rabadán Álvarez 1989: 250). El trío formado por Chapman, Cowley y Denham sentó las bases para las aportaciones más sistemáticas de John Dryden (1631-1700) y Alexander Pope (1688-1744) a finales del siglo XVII y la primera mitad del XVIII, período considerado por algunos críticos como la auténtica Edad de Oro de la traducción en Inglaterra (Amos 1920: 135) ${ }^{10}$.

Las reflexiones teóricas sobre traducción van acompañadas en The Arte por consideraciones de otro tipo, como valoraciones críticas por parte de George Puttenham hacia una traducción al inglés de La Eneida de Virgilio. A pesar de que Puttenham no desvela el nombre del traductor, se ha identificado a Gavin Douglas como el responsable de la misma (Morini 2006: 28). En el extracto siguiente, Puttenham arremete contra las elecciones léxicas en la traducción de Douglas que considera inapropiadas para aludir a un héroe de la talla de Eneas, y en un contexto tan elevado como el de la trama de La Eneida:

And as one, who translating certaine bookes of Virgils Aeneidos into English meetre, said that Aeneas was fayne to trudge out of Troy: which terme became better to be spoken of a beggar, or of a rogue, or a lackey: for so wee use to say to such maner of people, be trudging hence.

Another Englishing this word of Virgill [fato profugus] called Aeneas [by fate a fugitive] which was undecently spoken, and not to the Authours intent in the same word: (...) The same translatour when he came to these wordes: Infignem pietate virum, tot volvere casus tot adire labores compulit. Hee turned it thus, what moved Iuno to tugge so great a captaine as Aeneas, which word tugge spoken in this case is so undecent as none other coulde have bene devised... (Puttenham 1589/1970: 273-274)

La traducción de La Eneida al inglés también es mencionada brevemente en el capítulo xiI del libro segundo de The Arte, en el que se debate la manera de aplicar la métrica griega y latina a la poesía en lengua inglesa. Teniendo en mente que para Puttenham el traductor es un versificador, sería posible concebir este capítulo de The Arte como especialmente útil para los traductores al inglés de poesía en las lenguas clásicas. Al fin y al cabo, a pesar de que Puttenham no consideraba a los traductores lectores en potencia de su obra, muchos de los autores de poesía de la época, como Sir Thomas Wyatt o Henry Howard, Earl of Surrey, eran además traductores de poesía.

En otros casos, la traducción es la protagonista de algunas de las curiosas anécdotas palaciegas que Puttenham ocasionalmente cuenta a sus aristocráticos lectores para evitar que se aburran con la lectura de The Arte. Entre estas anécdotas lingüísticas o de traducción, destaca la acontecida al Emperador Carlos V con un embajador de Enrique viII, quien, según Puttenham, debido a una falta de dominio del castellano llamó al Emperador "Hombre el mas ingrato en el mondo», lo que desencadenó una desagradable escena en la que Carlos $\mathrm{V}$ le acaba instando a aprender mejor el idioma ${ }^{11}$. George Puttenham concluye el relato subrayando la importancia de utilizar los términos apropiados en cada situación:

\& all this inconvenience grew by misuse of one word, which being otherwise spoken \& in some sort qualified, had easily holpen all, \& yet th' Embassadour might sufficiently 
have satisfied his commission \& much better advaunced his purpose, as to have said for this word [ye are ingrate,] [...] so ye may see how a word spoken undecently, not knowing the phrase or proprietie of a language, maketh a whole matter many times miscarrie. (Puttenham 1589/1970: 270-271)

Finalmente, Puttenham deja ver sus dotes como traductor al verter al inglés ciertos versos latinos que utiliza para ejemplificar el uso de algunas figuras retóricas, o ilustrar alguno de sus comentarios sobre poesía. Asimismo, las vuelve a poner de manifiesto cuando en el tercero de los libros, Of Ornament, traduce al inglés los nombres en griego y en latín de más de cien figuras. Como se pondrá de manifiesto en las páginas que siguen, lejos de ofrecer unas traducciones convencionales, George Puttenham propone unas traducciones coloridas y visuales, acordes con las implicaciones o las consecuencias del uso de las figuras en el discurso.

\section{La práctica de la traducción en The Arte: la traducción al inglés de versos latinos}

Debido a que The Arte es ideado para ser leído por cortesanos nada duchos en las lenguas clásicas, prácticamente la totalidad de los ejemplos poéticos que no están escritos en lengua inglesa aparecen también traducidos al inglés ${ }^{12}$. Estas traducciones al inglés que se producen, principalmente, de versos en latín o de composiciones en italiano atribuidas a Petrarca, son llevadas a cabo por el propio George Puttenham en todos los casos salvo en uno. La excepción es el poema de Sir Thomas Wyatt «Set me whereas the sunne doth parch the greene» (Puttenham 1589/1970: 222), traducción al inglés de un soneto de Petrarca que no se reproduce en la obra de Puttenham ${ }^{13}$.

Un análisis de las traducciones al inglés de los versos latinos pone de relieve que Puttenham no seguía los principios de la traducción palabra por palabra, de los que, como se ha visto, los críticos franceses desconfiaban y los ingleses se desmarcaron por completo. Los extractos que aparecen a continuación dan buena fe de ello, pues si las traducciones al inglés de Puttenham se comparan con las traducciones literales al español que aparecen entre corchetes debajo de los versos latinos, se constata que el rétor inglés no sigue al pie de la letra los versos originales, sino que, más bien, se sirve de su idea general para llevar a cabo una creación poética propia. Así, las traducciones de Puttenham quedan impregnadas de un distintivo sabor inglés:

Multa renascentur quae iam cecidere cadent

Quae nunc sunt in honore vocabula si volet usus

Quem penes arbitrium est \& vis \& norma loquendi.

Which I have thus englished, but nothing with so good grace, nor so briefly as the Poet wrote:

Many a word ysalne shallest arise

And such as now bene held in hiest prise

Will fall as fast, when use and custome will

Onely umpiers of speach, for force and skill.

(Puttenham 1589/1970: 147-148)

Renacen muchas palabras que ya desaparecieron y desaparecen

las que ahora se tienen en consideración, si lo quiere el uso,

en poder del cual está el arbitrio, la esencia y la norma del lenguaje.

(Traducido por Pastor Andrés ${ }^{14}$ ) 
(2) Scire tuum nihil est nisi te scire, hoc sciat alter.

Which I have turned into English, not so briefly, but more at large of purpose the better to declare the nature of the figure: as thus,

Thou weenest thy wit nought worth if other weet it not

As well as thou thy selfe, but o thing well I wot,

Who so in earnest weenes, he doth in mine advise,

Shew himselfe witlesse, or more wittie than wise.

(Puttenham 1589/1970: 204)

Tu saber nada es, a no ser que otro sepa que tú sabes esto

(Traducido por Pastor Andrés)

(3)

Tantae a molis erat Romanam condere gentem.

In English thus:

So huge a peece of worke it was and so hie,

To reare the house of Romane progenie.

(Puttenham 1589/1970: 216)

¡De tan gran esfuerzo era el fundar el pueblo romano!

(Traducido por Pastor Andrés)

Como se aprecia a simple vista, las traducciones de Puttenham están lejos de ser calcos de los originales, pues, para empezar, no hay una correspondencia entre el número de versos de los originales en latín con sus traducciones al inglés: los tres versos latinos del primer ejemplo se convierten en cuatro en la traducción de Puttenham; en el segundo ejemplo, de un solo verso latino Puttenham produce cuatro en inglés; y en el tercero de los ejemplos, cada verso en latín es traducido por dos en lengua inglesa. Esto significa que Puttenham realmente toma como punto de partida los versos latinos para componer los suyos propios, matizando o elaborando las ideas de los originales según su conveniencia. A modo de ilustración, bastará considerar las transformaciones en términos de contenido del último de los versos latinos citados más arriba:

(4) Tantum relligio potuit suadere malorum.

In English thus:

Lo what an outrage, could cause to be done,

The peevish scruple of blinde religion.

(Puttenham 1589/1970: 217)

¡Tantas cosas malas ha podido causar la religión!

(Traducido por Pastor Andrés)

Mientras que el verso latino se refiere a la religión en general, Puttenham matiza la idea de religión con una serie de modificadores que aparecen antepuestos a ella: «the peevish scruple of blinde religion». En otras palabras, no es la religión lo que puede ser causa de sucesos desafortunados, sino «la fastidiosa ansiedad de la ciega religión». Es más que probable que esta distinción entre religión en términos generales y «ciega religión» fuera necesaria en un momento histórico en el que la religión era un tema candente no sólo en Inglaterra sino en toda Europa al que estaban íntimamente ligadas muchas de las turbulencias políticas de la época.

Las traducciones al inglés arriba citadas tienen en común que están estructuradas en forma de pareado, que es el tipo de verso que posteriormente Denham, y tras 
él John Dryden, popularizarían en sus traducciones. El pareado ha sido visto como una estrategia estrella para lograr en las traducciones de poesía un discurso fluido que produce una ilusión de transparencia, y que oculta que el texto que se lee tiene el estatus de traducción (Venuti 1995: 63). De esta forma, como también se pondrá de manifiesto en el análisis de la traducción de los nombres de las figuras retóricas, George Puttenham, al igual que muchos de sus colegas traductores en la época isabelina, otorga un distintivo sabor inglés a sus traducciones acercándolas al lector del siglo xvi. Como subraya F. O. Matthiessen, ésa era precisamente una de las características más significativas de la labor de los traductores isabelinos:

[...] his [the Elizabethan translator's] diction was racy and vivid, thronged with proverbial phrases, the slang of the streets, bold compounds, robust Saxon epithets, and metaphors drawn from English ports and countryside. The structure of his sentences reveals the growing tendencies of the time - the passionate delight in fullness of expression, the free use of doublets and alliteration, the building up of parallel constructions for the sake of rhythm. (Matthiessen 1931: 4)

Esta búsqueda de los traductores por lograr una traducción fluida que produjera la ilusión de leer un original y no una traducción, y que se vio impulsada en el siglo XVII, está en los orígenes de la noción de la invisibilidad del traductor desarrollada por Lawrence Venuti (1995).

En el caso particular de The Arte, también hay que tener en cuenta a la hora de considerar las traducciones al inglés de los versos latinos que Puttenham realmente empleaba la traducción como un instrumento pedagógico. Al fin y al cabo, los versos latinos eran ejemplos que ilustraban los contenidos teóricos de The Arte. Por tanto, si los mismos ejemplos acababan constituyendo un problema para unos lectores no versados en las lenguas clásicas, la finalidad de los mismos se veía truncada. En otras palabras, Puttenham podría haber presentido que si no acercaba la traducción de los versos latinos a los lectores, sus ejemplos, aun estando en inglés, se convertirían en un obstáculo en lugar de suponer un apoyo a la comprensión de la teoría.

Esta preocupación por la claridad se refleja en que, en algunos casos, tras traducir un reducido número de versos del latín al inglés, Puttenham proporciona, además, una paráfrasis en prosa de su traducción con el objetivo de disipar cualquier duda que pudieran seguir teniendo los lectores. Curiosamente, se sirve de este procedimiento cuando sus traducciones muestran un mayor apego al original, como se observa en los versos que se citan a continuación:

Nocte pluit tota, redeunt spectacula mane

Divisum imperium cum Iove Caesar habet.

Which I have thus Englished,

It raines all night, early the snewes returne

God and Caesar, do raigne and rule by turne.

As much as to say, God sheweth his power by the night raines. Caesar his magnificence by the pompes of the day.

(Puttenham 1589/1970: 55)

Llueve durante toda la noche $y$, por la mañana, vuelven los espectáculos; César tiene el imperio dividido con Júpiter. 
(6)

Veni, vidi, vici

I came, I saw, I overcame.

Meaning thus I was no sooner come and beheld them but the victorie fell on my side.

(Puttenham 1589/1970: 175)

Vine, vi, vencí

(Traducido por Pastor Andrés)

Claudite iam rivos puere sat prata biberunt.

Which I English thus:

Stop up your streames (my lads) the medes have drunk ther fill.

As much to say, leave of now, yee have talked of the matter inough:

(Puttenham 1589/1970: 187)

Cerrad ya los arroyos, niños, suficiente bebieron los prados.

(Traducido por Pastor Andrés)

El empleo de la paráfrasis en prosa tras la traducción al inglés se convierte, así, en la manera que tiene Puttenham de asegurarse de que los lectores interpretarían correctamente el significado último de sus ejemplos. La voluntad de Puttenham por adecuar The Arte al perfil de sus lectores es además un factor decisivo para entender las razones que le llevaron a traducir los nombres de las figuras retóricas al inglés, y los motivos por los que los tradujo de la manera en que lo hizo.

\section{La práctica de la traducción en The Arte: la traducción al inglés de los nombres de las figuras retóricas}

El número total de figuras retóricas mencionadas en el libro tercero «Of Ornament» asciende a ciento dos, a las que hay que sumar seis subcategorías de figuras, y un total de quince tipos de vicios o faltas en la escritura que yo también incluyo dentro de la noción de figura retórica ${ }^{15}$. Puttenham hace una distinción en tres grandes grupos de figuras («auricular figures», «sensable figures», $y$ «sententious figures») por medio de dos criterios diferentes: el perfil de la persona que las utiliza normalmente (poetas, oradores, $\mathrm{o}$ ambos), y el aspecto al que sus propiedades persuasivas apelan (el sonido, o el pensamiento). De esta manera, las figuras auditivas («auricular figures») son las propias del poeta y resultan eficaces por una cuestión de sonido; las figuras juiciosas («sensable figures») apelan al pensamiento y son tan propias del poeta como del orador; y las figuras sentenciosas o retóricas («sententious figures»), son utilizadas principalmente por el orador, y deben su éxito tanto a su sonido como a su capacidad de persuasión a través del pensamiento. La clasificación propuesta por Puttenham es altamente novedosa ya que «se aparta de la tradición retórica al clasificar según la recepción antes que por criterios formales», lo cual la convierte en un hecho único en el período renacentista (Plett 1999: 438).

La estructura de las explicaciones de las figuras es casi siempre la misma: Puttenham ofrece una breve descripción del significado de la figura, su nombre griego o latino o ambos, una traducción o traducciones al inglés de dicho nombre, y uno o varios ejemplos del uso de la figura en composiciones poéticas. Lo curioso de las traducciones de los nombres de las figuras es que en ocasiones se llevan a cabo utilizando figuras retóricas como estrategias de traducción. En otras palabras, Puttenham traduce algunos nombres de figuras por medio de alegorías, y otros a través de distintos símiles. 


\subsection{Traducciones fundamentadas en la alegoría «comportamiento del cortesano» = «ornamento poético»}

En The Arte se pueden distinguir hasta cuarenta y cinco figuras cuya traducción al inglés se basa en una alegoría por la que el comportamiento del cortesano equivale a ornamento poético. Esto tiene como consecuencia que, cuando George Puttenham se enfrenta a la traducción al inglés de sus nombres, los traduce como si tuviera que definir en una palabra el tipo de persona que lleva a cabo en la vida real lo que las figuras hacen en el discurso. Esto es lo que Plett y Heath denominan un procedimiento de traducción basado en la invención de nomina agentis para las figuras (Plett y Heath 1983: 607). Por ejemplo, la figura del antíteton, que implica una discrepancia entre dos pensamientos, es traducida como The Quarreller, el polemista. Puttenham argumenta tal traducción recordando sus supuestos tiempos como estudiante en Oxford ${ }^{16}$ : «for so be al such persons as delight in taking the contrary part of whatsoever shalbe spoken: when I was a scholler in Oxford they called every such one Iohannes ad oppositum» (Puttenham 1589/1970: 210). Así, mediante este procedimiento «las formas estilísticas indican roles sociales» $\mathrm{y}$ «los roles sociales se muestran en ciertas categorías estilísticas» (Plett 1999: 435). A continuación se enumeran, por orden de aparición en The Arte, las figuras traducidas a través de este original procedimiento de traducción.

CUADRO 1

Traducciones fundamentadas en la alegoría "comportamiento del cortesano»: «ornamento poético»

\begin{tabular}{|l|l|l|}
\hline Nombre de la figura & Traducción Puttenham & Traducción al español \\
\hline Prozeugma & Ringleader & Cabecilla \\
Mezozeugma & Middlemarcher & Medianero \\
Hipozeugma & Rerewarder & El farolillo rojo \\
Hipozeuxis & The substitute & El sustituto \\
Prolepsis & The propounder or The explaner & El exponedor o explicador \\
Hipérbaton & Trespasser & Trasgresor \\
Histeroproterón & The preposterous & El preposterador \\
Epíteto & The qualifier & El caracterizador \\
Metonimia & The misnamer & El mal denominador \\
Antonomasia & The surnamer & El apellidador \\
Onomatopeya & The new namer & El inventor de nombres \\
Metalepsis & The farrafet & El rebuscado \\
Énfasis & The renforcer & El reforzador \\
Lítote & The moderator & El moderador \\
Paradiástole & The curry-favell & El hipócrita \\
Meiosis & The disabler & El difamador \\
Tapinosis & The abbaser & El degradador \\
Hipérbole & The over-reacher or The loud lyar & El fantasmón o El trolero \\
Ploqué & The doubler & El duplicador \\
Prosonomasia & The nicknamer & El apodador \\
Insulto & The disdainefull or The reprochfull & El desdeñador o El reprochador o \\
& or Scorner & El vilipendiador \\
Antíteton & The quarreller & El disputador \\
Erotema & The questioner or Inquisitive & El preguntador o Inquiridor \\
Metanoia & The penitent or Repentant & Figura del penitente o del \\
Antanagoge & The recompencer & arrepentido \\
Aúxesis & The avancer & El compensador \\
Diálisis & The dismembrer & El aumentador \\
Merismo & The distributer & El despedazador \\
Aporáa & The wondrer & El distribuidor \\
Parisía & The doubtful & El maravillado \\
\hline
\end{tabular}




\begin{tabular}{|l|l|l|} 
Anacoenosis & The impartener & El indagador \\
Orismo & The definer of difference & El definidor de diferencias \\
Procatalepsis & The presumptuous & El anticipador \\
Paralipsis & The passager & El transeúnte \\
Parécbasis & The stragler & El rezagado \\
Expeditio & The speedie dispatcher & El despachante diligente \\
Dialogismo & The right reasoner & El buen razonador \\
Gnome/Sententia & The director or The sage sayer & El director o El hablante sabio \\
Sinatrismo & The heaping figure & El colector \\
Cacosínteton & The misplacer & El desordenado \\
Cacénfaton & The unshamefast & El truhán \\
Acirón & The uncouthe & El grosero \\
Periergía & The curious & El indagador \\
Anfibología & The ambiguous & El ambiguo \\
\hline
\end{tabular}

La utilización de la alegoría para traducir las figuras retóricas arriba mencionadas se ha considerado un hecho "excepcional en la retórica estilística isabelina» (Plett 1999: 435). El empleo de esta estrategia demuestra una vez más la voluntad de Puttenham por adecuar las características de The Arte al perfil de sus lectores cortesanos. Asimismo, refleja el impacto que tuvo en Inglaterra la traducción en 1561, por parte de Thomas Hoby, de Il Cortegiano de Castiglione ${ }^{17}$. Daniel Javitch ha estudiado en profundidad las relaciones entre la obra de Puttenham, a la que considera un manual de conducta social, con la de Castiglione, llegando a conclusiones como las que refiere el extracto siguiente:

the prescriptions of the Cortegiano substantially agree with the Arte's poetic directives and the standards of conduct on which they are based. This effective collocation of the Cortegiano and the Arte can be taken as further proof of the impact of the Italian book on English court manners and aesthetics in general. It certainly suggests to what extent the Elizabethan courtly code embodied in Puttenham's poetics conformed to Castiglione's. (Javitch 1972: 871)

Desde este punto de vista, Puttenham traslada a la poesía el código cortesano descrito por Castiglione, lo cual hace que el ornatus no pueda ser concebido «como un mero accesorio estético», sino como «un constituyente esencial de la literatura cortesana en la misma medida en que una magnífica vestimenta es una parte integral de la existencia del cortesano» (Plett 1999: 435). Por ello, las figuras adquieren en The Arte un valor añadido.

\subsection{Traducciones basadas en comparaciones}

Si la alegoría subyace a las traducciones arriba mencionadas, el símil o comparación está en la base de las traducciones de otros nombres de figuras. Las traducciones basadas en la comparación remiten a objetos o hechos de la vida cotidiana que para Puttenham ilustran las implicaciones del uso de una determinada figura. Por ejemplo, Puttenham traduce como The Rebound (el rebote) la figura de la anaclasis, que recordemos «consiste en repetir en un diálogo, y por distintos interlocutores, la misma o las mismas palabras con sentido o intención diferente, pero habitual y posible en cada uno de ellos» (Álvarez 1989: 15). George Puttenham explica en los siguientes términos las razones de tal traducción: «we may call [this figure] the Rebound, alluding to the tennis ball which being smitten with the racket reboundes backe againe, and where the last figure before played with two wordes somewhat like, this playeth with one word written all alike but carrying divers sences» (Puttenham 1589/1970: 207). 
En otras palabras, la traducción del nombre de la figura de la anaclasis se basa en el símil que compara la repetición que lleva consigo el empleo de tal figura con el rebote de la pelota en el juego del tenis.

Puttenham retoma el deporte como término de comparación para la traducción de omoioteleton ${ }^{18}$, «homofonía que se produce entre dos o más palabras de la misma categoría gramatical a partir de la última vocal acentuada y siempre que tales vocablos vayan muy cercanos» (Álvarez 1989: 98). En este caso, es el campo semántico del tiro con arco el que sirve de inspiración a Puttenham:

we call this figure following the originall, the [like loose] alluding to th' Archers terme who is not said to finish the feate of his shot before he give the loose, and deliver his arrow from his bow, in which respect we use to say marke the loose of a thing for marke the end of it. (Puttenham 1589/1970: 174)

En total son nueve los nombres de las figuras que Puttenham traduce por el procedimiento del símil. El cuadro siguiente los representa de manera esquemática:

CUADRO 2

\section{Traducciones basadas en comparaciones}

\begin{tabular}{|l|l|l|}
\hline Nombre de la figura & Traducción Puttenham & Traducción al español \\
\hline Hipálage & Changeling & Cambiazo \\
\hline Omoioteleton & Like loose & El mismo final \\
\hline Endíadis & The figure of Twynnes & La figura de los mellizos \\
\hline Epanalepsis & The eccho sound, or The flow return & El Eco o El retorno fluido \\
\hline Epizeuxis & Underlay or The cuckow-spell & El soporte o el canto del cuco \\
\hline Sineciosis & The Crosse-couple & El cruzamiento \\
\hline Anaclasis & The Rebound & El rebote \\
\hline Epímona & The Love-burden & Estribillo \\
\hline Conmoración & The figure of abode & La figura del domicilio \\
\hline
\end{tabular}

Muchas de las comparaciones en las que se basan las traducciones enumeradas arriba sorprenden por su originalidad. Este es el caso de sineciosis, figura por la que se acercan en el discurso dos palabras con significados opuestos. Puttenham la traduce al inglés como The Crosse-couple (el cruzamiento), y explica el porqué de su decisión de la siguiente manera: «because it takes me two contrary words, and tieth them as it were in a paire of couples, and so makes them agree like good fellowes, as I saw once in Fraunce a wolfe coupled with a mastisse, and a foxe with a hounde» (Puttenham 1589/1970: 206). En este caso, el símil, claramente indicado a través del uso de as, se resume en que unir un par de antónimos es como cruzar dos animales de especies diferentes.

De igual manera, la traducción de hipálage por changeling también destaca por su originalidad. Recordemos que hipálage consiste en una atribución «a un sustantivo un adjetivo que significativamente no le corresponde pero que resulta más expresivo» (Álvarez 1989: 77), y que el término changeling se utilizaba en la segunda mitad del siglo XVI para aludir al cambiazo de un niño pequeño por otro. Teniendo esto en mente, es posible entender la explicación que Puttenham da de tan sorprendente traducción: 
The Greekes call this figure [Hipallage] the Latins Submutatio, we in our vulgar may call him the [underchange] but I had rather have him called the [Changeling] nothing at all swerving from his originall, and much more aptly to the purpose, and pleasanter to beare in memory: specially for our Ladies and pretie mistresses in Court, for whose learning I write, because it is a term often in their mouthes, and alluding to the opinion of Nurses, who are wont to say, that the Fayries use to steale the fairest children out of their cradles, and put other ill favoured in their places, which they called changelings, or Elfs: so, if ye mark, doeth our Poet, or maker play with his wordes, using a wrong construction for a right, and an absurd for a sensible, by manner of exchange. (Puttenham 1589/1970: 172-173)

En definitiva, Puttenham elige el término changeling simplemente porque les resultaba más familiar a las damas de la Corte, a quienes The Arte también iba dirigido. Con toda certeza, sería menos problemático para una cortesana inglesa de finales del xvi recordar un término que ya utilizaba en su vida diaria, que memorizar la voz griega.

Puttenham era consciente de que The Arte era susceptible de ser muy criticado por sus contemporáneos debido precisamente a estas traducciones, pues ello significaba proponer una alternativa en lengua inglesa a los nombres de las figuras retóricas, los pilares fundamentales de la nomenclatura de la elocutio clásica. Los detractores no podrían ser sino grandes conocedores de la retórica y las lenguas clásicas con una cierta mentalidad conservadora. El rétor inglés se adelanta a las críticas afirmando que lo que le ha movido a llevar a cabo las traducciones ha sido su deseo por facilitar a sus lectores cortesanos el aprendizaje de las figuras retóricas. El autor de The Arte lo expresa de la siguiente manera:

Againe if to avoid the hazard of this blame I should have kept the Greek or Latin still it would have appeared a little too sholasticall for our makers, and a peece of worke more fit for clerkes then for Courtiers for whose instruction this travaile is taken: and if I should have left out both the Greeke and Latine name, and put in none of our owne neither: well perchance might the rule of the figure have bene set downe, but no convenient name to hold him in memory. It was therefore expedient we devised for every figure of importance his vulgar name, and to joyne the Greeke of Latine originall with them; after that sort much better satisfying aswel the vulgar as the learned learner, and also the authors owne purpose, which is to make of a ruderimer, a learned and a Courtly Poet. (Puttenham 1589/1970: 158)

Previendo que sus traducciones podrían ser tachadas de ridículas por aquellos lectores versados en las lenguas clásicas o familiarizados con la disciplina de la retórica, Puttenham se adelanta de nuevo a las críticas reconociendo que sus traducciones pueden al principio resultar extrañas simplemente por la novedad, pero que con el tiempo el sentimiento de extrañeza desaparece:

And in case any of these new English names given by me to any figure, shall happen to offend I pray that the learned will beare with me and to thinke the straungenesse thereof proceedes but of noveltie and disaquaintance with our eares, which in processe of tyme, and by custome will frame very well. (Puttenham 1589/1970: 157)

El hecho de que Puttenham justificara con tanto ahínco sus traducciones frente a las posibles críticas de los expertos en retórica y lenguas clásicas, indica el poder y el reconocimiento académico que éstos últimos ostentaban en la época en que se publicó The Arte. De otro modo no se podría comprender por qué el autor de la 
traducción de unos términos aislados en un manual de poética/retórica para cortesanos necesitara justificar una y otra vez los motivos por los que los traduce.

\section{Conclusiones}

Si la hipótesis principal de la que partía el presente estudio era que existía la posibilidad de que la definición de traducción poética proporcionada por George Puttenham no se ajustara a la realidad de la práctica de la misma a lo largo de The Arte, en este apartado de conclusiones finales es posible constatar que el análisis llevado a cabo en el cuerpo del trabajo sobre las distintas manifestaciones de la traducción en dicha obra confirman la sospecha inicial de discrepancia entre teoría y práctica de la traducción en The Arte.

Puttenham abría su manual de retórica con una definición de poesía y de poeta que a su vez utilizaba para definir a la traducción y al traductor. Así, afirmaba que el poeta se caracterizaba por ser un creador (a maker), al elegir tanto el contenido de su composición poética como la forma que ésta debería adoptar. El traductor, en cambio, se limitaba a poner en práctica sus habilidades de versificación, puesto que el contenido que debía verter a otra lengua ya le era dado por el modelo de la composición original. De esta manera, Puttenham establecía una ecuación entre creador, poeta, y facultades creativas; y versificador, traductor, y copia carente de originalidad o creatividad. Como consecuencia de ello, se desproveía a la labor del traductor de cualquier reconocimiento de creatividad, relegando su trabajo a una mera actividad de cálculo relacionada con los aspectos formales de la poesía. Incluso a pesar de reconocer que la poesía en ocasiones también podía ser imitativa, la imitación propia de la traducción continuaba localizándose por debajo de ella.

El análisis de la práctica de la traducción en The Arte pone de relieve, sin embargo, que el traductor es mucho más que un simple versificador, pues si hay un rasgo que destacar de las traducciones de George Puttenham es su alta creatividad. Lejos de preocuparse por meros rasgos formales, George Puttenham tiene como objetivo prioritario a la hora de traducir al inglés tanto los versos latinos, como los nombres de las figuras retóricas, lograr una adecuación entre sus traducciones y el perfil y expectativas de su público lector, constituido, en su gran mayoría, por cortesanos ingleses de finales del siglo xvi. En otras palabras, George Puttenham antepone lo que para Jean Delisle sería la función pedagógica de sus traducciones (Delisle 2003: 14) a cualquier otro tipo de consideraciones, por lo que sus traducciones están condicionadas por completo por su voluntad de hacerlas fácilmente entendibles a sus lectores.

La falta de concordancia entre las «reflexiones teóricas» de George Puttenham con respecto a la manera en que ejecuta sus traducciones en distintos momentos de The Arte dista de ser un hecho insólito en esta época de la historia de la traducción en Inglaterra, y confirma la discrepancia advertida por Massimiliano Morini entre los postulados sobre traducción en los prefacios a las traducciones de este periodo, y el proceder real de los traductores a la hora de verter textos al inglés. Para Morini, la existencia de este abismo entre teoría y práctica tiene que ver con el hecho de que el siglo XVI en Inglaterra es un periodo de transición en el que conviven ideas medievales sobre la traducción con otras modernas, de corte humanista, que exigen una mayor atención hacia la organización retórica del texto a traducir (Morini 2006: vii). 
En definitiva, si algo muestra el presente análisis de The Arte es que Lieven D'hulst estaba en lo cierto: las obras de retórica sin duda se muestran como documentos de gran valía para los especialistas en historia de la traducción, al guardar celosamente datos que ayudan a vislumbrar con mayor claridad la evolución de la teoría y la práctica de la traducción a lo largo de la historia.

\section{NOTAS}

1. Puttenham, George (1589/1970): The Arte of English Poesie. In: Gladys Doidge Willcock y Alice WALKer, eds. Cambridge: Cambridge University Press. Diversos autores han reconocido límites difusos entre las disciplinas de la retórica y la poética durante el Renacimiento (Kristeller 1979: 251; Kahn 1985: 38; Clark 2004: 5-7). La naturaleza híbrida de The Arte of English Poesie se refleja en el hecho de que Heinrich F. Plett lo clasifica como una poética (Plett 1995: 225), al tiempo que reconoce al tercer libro de The Arte, «Of Ornament», como un ejemplo de retórica estilística (Plett 1995: 136). De igual forma, L.D. Green y J.J. Murphy incluyen a este tercer libro en su catálogo bibliográfico de retóricas (Green y Murphy 2006: 350), y J.J. Murphy considera a George Puttenham uno de los veinte miembros del exclusivo «canon de retóricos» (Murphy 1999). Por todo ello, a lo largo del presente artículo me referiré a la obra de Puttenham como un tratado de poética/retórica.

2. Para un inventario bastante completo de las traducciones llevadas a cabo en Gran Bretaña en esta época, consultar Ebel (1967) y Van Hoof (1991: 122-144).

3. Lisa Gim (1998) analiza estos poemas de Puttenham poniéndolos en relación con The Arte, y prestando especial atención a la función que desempeña en ellos la alegoría.

4. A pesar de que la retórica clásica hace una distinción entre figuras y tropos, George Puttenham solamente habla de figuras.

5. En la Inglaterra del siglo xvi, maker era una voz común para referirse a poet, al igual que lo fue el vocablo makar, con obvias conexiones etimológicas, en Escocia. El propio Sir Philip Sidney, en su An Apology for Poetry, hablando sobre la etimología del término poet, dice lo siguiente: «[Poet] cometh of this word poiein, which is, to make: wherein, I know not whether by luck or wisdom, we Englishmen have met with the Greeks in calling him a maker» (Sidney 2002: 84).

6. La filosofía neoplatónica distinguía tres tipos de poesía, que se correspondían con los tres tipos de facultades del alma: fantasía, que trataba de las apariencias; razón, que buscaba las causas detrás de las apariencias; y mente, capaz de una visión directa de la verdad (Preminger y Brogan 1993: 912). Para más información acerca de la historia y el impacto del neoplatonismo en Inglaterra, ver Cassirer (1953).

7. Paul Chavy (1988) ofrece una lista bastante completa de traductores franceses de tanto la Edad Media como del Renacimiento en la que también se incluyen las traducciones que llevaron a cabo y su fecha de impresión. Para más información sobre la traducción en Francia en el siglo xvi, ver Suso López (1995: 115-122). Por otra parte, para un estudio sobre las primeras reflexiones teóricas acerca de la traducción en el contexto del humanismo europeo de la segunda mitad del siglo XV, ver Norton (1981).

8. La imagen del traductor como pintor también es un tema recurrente en los escritos de los traductores neoclásicos ingleses, quienes describían la labor de traducción en términos pictóricos al reconocer la existencia de un similar tipo de mímesis entre la pintura y la traducción (Steiner 1975: 35).

9. Theo Hermans también es autor de otro estudio que toca el tema de la relación entre imitación y traducción en el Renacimiento en Inglaterra (Hermans 1992).

10. Para otros autores, en cambio, el siglo xvi brilló más que el xvir en lo tocante a la traducción (Savory 1957: 41).

11. Según constata Jason Powell (2005: 175), el embajador en cuestión era, nada más y nada menos, que el célebre Thomas Wyatt. Sin embargo, para Powell es probable que el suceso no fuera tal como lo narra Puttenham, quien podría haber retocado considerablemente la conversación original entre Carlos V y Wyatt: «Puttenham's account was clearly second-hand; it is so heavily corrupted that it may even have emerged from rumours outside of court» (Powell 2005: 176).

12. Los únicos versos que no aparecen traducidos al inglés son los versos latinos localizados en las páginas 12-15, en el Capítulo VII del Libro I («How in the time of Charlemaine and many yeares after him the Latine Poetes wrote in ryme»); en la página 22, Libro I, Capítulo VIII, en donde se reproducen tres versos a propósito de la negativa del Emperador Octavio a quemar los libros de 
La Eneida tal y como había pedido en su testamento Virgilio; y en la página 55, Libro I, Capítulo XXVII, en donde se reproducen unos versos escritos por el autor de La Eneida.

13. Este procedimiento de incluir una traducción identificándola como traducción pero sin acompañarla de su original correspondiente, es utilizado en otras dos ocasiones en The Arte cuando los originales son de una considerable longitud. Así, no se reproduce ni la composición poética de doce versos en griego clásico escrita por el filósofo Crates acerca de las dificultades y sinsabores de la vida del hombre (Puttenham 1589/1970: 205), ni otro poema de Petrarca, traducido esta vez por Puttenham (Puttenham 1589/1970: 241-242).

14. Héctor Felipe Pastor Andrés es el autor de las traducciones al español de los versos latinos.

15. Al hablar de subcategorías me refiero a que hay algunas figuras retóricas que tienen subtipos. Este es el caso de, por ejemplo, zeugma, categoría más general dentro de la que se incluye prozeugma, mezozeugma, e hypozeugma. En lo tocante a los vicios o faltas en la escritura, hay diversidad de opiniones: Fernando Marcos Álvarez (1989) y La Rue van Hook (1914), por ejemplo, también los consideran figuras retóricas, mientras que Edward Corbett (1971: 610) o Gideon Burton, autor de la página web $<$ http://humanities.byu.edu/rhetoric/silva.htm $>$, los excluyen de la lista de figuras.

16. Realmente, George Puttenham no estudió en Oxford (Lee 2001: 65). Esta es una de las contradicciones entre la vida de George Puttenham y la que dice tener el autor de The Arte que llevaron a críticos como B. M. Ward a cuestionar la autoría del texto por parte de Puttenham (Ward 1925). En la actualidad, la autoría de Puttenham está fuera de toda duda. Para más información sobre el anonimato de y en The Arte, consultar (North 1999).

17. Para un estudio en profundidad sobre la traducción de la obra de Castiglione al inglés, y su impacto en la sociedad inglesa, consultar la obra de F. O. Matthiessen (1931: 8-53).

18. El término omoioteleton también puede encontrarse escrito en español de las siguientes tres formas: homoioteleton, homoteleuton, y homeoteleuton.

\section{REFERENCIAS BIBLIOGRÁFICAS}

Álvarez, Fernando Marcos (1989): Diccionario práctico de recursos expresivos (figuras y tropos). Cáceres: Universidad de Extremadura.

Amos, Flora Ross (1920): Early Theories on Translation. Nueva York: Columbia University Press. Arduini, Stefano (2000): Prolegómenos a una teoría general de las figuras. Murcia: Universidad de Murcia.

Bassnett-McGuire, Susan (1980): Translation Studies. London: Methuen and Co.

Burton, Gideon (Actualizado el 26 de febrero de 2007): The Forest of Rhetoric. Silva rhetoricae. Consultado el 1 de febrero de 2010, <http://humanities.byu.edu/rhetoric/>.

Cassirer, Ernst (1953): The Platonic Renaissance in England. (Traducido por James P. PetteGROVE) Edinburg: Nelson.

Chavy, Paul (1988): Traducteurs d'autrefois, Moyen Age et Renaissance: Dictionnaire des traducteurs et de la littérature traduite en ancien et moyen français (842-1600). París: ChampionSlatkine.

Chico Rico, Francisco (2002): La teoría de la traducción en la teoría retórica. Revista de retórica y teoría de la comunicación. 3:25-40.

ClARK, Donald Lemen (2004): Rhetoric and Poetry in the Renaissance. Nueva York: Kessinger Publishing.

Conbett, Edward P.J. (1971): Classical Rhetoric for the Modern Student. Nueva York: Oxford University Press.

Delisle, Jean (2003): La historia de la traducción: Su importancia para la traductología y su enseñanza mediante un programa didáctico multimedia y multilingüe. Sendebar. 14:5-16.

D'Hulst, Lieven (1995): Pour une historiographie des théories de la traduction: questions de méthode. TTR. 1(8):13-33.

Du Bellay, Joachim (1549/1997): La deffence et illustration de la langue françoyse. París: Société des Textes Français Modernes.

Ebel, Julia G. (1967): A numerical survey of Elizabethan translations. The Library, A Quarterly Journal of Bibliography. 22:104-127. 
García Peinado, Miguel A. (2002): Acerca de la traducción y la imitación en Du Bellay. In: Vicente López Folgado, ed. Sensus de sensu: Estudios filológicos de traducción. Córdoba: Universidad de Córdoba, 105-113.

Gim, Lisa (1998): Blasoning "the Princesse Paragon": The workings of George Puttenham's "False Semblant" in his Partheniades to queen Elizabeth. Modern Language Studies. 3-4(28):75-89.

Goyet, Francis, ed. (1990): Traités de poétique et de rhétorique de la Renaissance. París: Librairie Générale Française.

Green, Lawrence D. y Murphy, James J. (2006): Renaissance Rhetoric Short-Title catalogue 1460-1700. Hants: Ashgate.

Hermans, Theo (1985): Images of translation. Metaphor and imagery in the Renaissance discourse on translation. In: Theo Hermans, ed. The Manipulation of Literature: Studies in Literary Translation. Nueva York: St. Martin's Press, 103-135.

Hermans, Theo (1992): Renaissance translation between literalism and imitation. In: Herausgegeben von Harald KitTeL, ed. Geschichte, System, Literarische Übersetzung = Histories, systems, literary translations. Berlín: Schmidt, 95-116.

Howatson, M.C. y Chilvers, Ian, eds. (1993): The concise Oxford companion to classical literature. Oxford/Nueva York: Oxford University Press.

Javitch, Daniel (1972): Poetry and court conduct: Puttenham's Arte of English Poesie in the light of Castiglione's Cortegiano. MLN. 7(87):865-882.

Kann, Victoria (1985): Rhetoric, Prudence and Skepticism in the Renaissance. Ithaca: Cornell University Press.

Kristeller, Paul Oskar (1979): Renaissance Thought and its Sources. Nueva York: Columbia University Press.

Kristeller, Paul Oskar (1999): La retórica en la cultura medieval y renacentista. (Traducido por Gaspar Garrote Bernal) In: James J. Murphy, ed. La elocuencia en el Renacimiento: Estudios sobre la teoría y la práctica de la retórica renacentista. Madrid: Visor, 11-31.

LeE, Sidney (2001): Puttenham, George. In: Sidney LeE, ed. Dictionary of National Biography. Vol. XLVII. Boston: Adamant Media Corporation, 64-67.

Mack, Peter (2002): Elizabethan Rhetoric: Theory and Practice. Cambridge/Nueva York: Cambridge University Press.

Matthiessen, Francis Otto (1931): Translation. An Elizabethan Art. Cambridge: Harvard University Press.

Morini, Massimiliano (2006): Tudor Translation in Theory and Practice. Aldershot: Ashgate Publishing Company.

Murphy, James J. (1999): Mil autores olvidados: Panorama e importancia de la retórica en el renacimiento. (Traducido por Gaspar Garrote Bernal) In: James J. Murphy, ed. La elocuencia en el Renacimiento: Estudios sobre la teoría y la práctica de la retórica renacentista. Madrid: Visor, 33-51.

North, Marcy L. (1999): Anonymity's revelations in The Arte of English Poesie. Studies in English Literature. 1500-1900. 1(39):1-18.

Norton, Glyn P. (1981): Humanist foundations of translation theory (1400-1450): A study in the dynamics of word. Canadian Review of Comparative Literature. 8(2):173-203.

Oliver, María (1995): Retórica y traducción. In: Francisco Lafarga, Albert Ribas y Mercedes TricÁs, eds. La traducción: Metodología, historia, literatura: Ámbito hipanofrancés. Barcelona: Promociones y Publicaciones Universitarias, 23-26.

Plett, Heinrich F. (1995): English Renaissance Rhetoric and Poetics: A Systematic Bibliography of Primary and Secondary Sources. Leiden: E.J. Brill.

Plett, Heinrich F. (1999): Lugar y función del estilo en la poética renacentista. (Traducido por J. Ignacio Díez Fernández) In: James J. Murphy, ed. La elocuencia en el Renacimiento: Estudios sobre la teoría y la práctica de la retórica renacentista. Madrid: Visor, 421-443.

Plett, Heinrich F. y Heath, Peter (1983): Aesthetic constituents in the courtly culture of Renaissance England. New Literary History. 3(14):597-621. 
Powell, Jason (2005): Puttenham's Arte of English Poesie and Thomas Wyatt's diplomacy. Notes and Queries. 52(2):174-176.

Preminger, Alex y Brogan, Terry V. F., eds. (1993): The New Princeton Encyclopedia of Poetry and Poetics. Princeton: Princeton University Press.

Rabadán Álvarez, Rosa (1989): Apuntes de teoría de la traducción en la Inglaterra del s. XVII. In: Julio César SANtoyo, ed. Fidus interpres: Actas de las Primeras Jornadas Nacionales de Historia de la Traducción. León: Universidad de León/Diputación Provincial de León, 249-254.

Rener, Frederick M. (1989): Interpretatio: Language and translation from Cicero to Tytler. Amsterdam: Rodopi.

SAvory, Theodore (1957): The Art of Translation. London: Jonathan Cape.

Sidney, Philip (2002): An Apology for Poetry: Or, the Defence of Poesy. In: Geoffrey ShePherd, ed. Manchester: Manchester University Press.

Steiner, Thomas Robert (1975): English Translation Theory: 1650-1800. Assen: Van Gorcum.

Suso López, Javier (1995): La conception de la traduction en France au XVI ${ }^{e}$ siècle. In: Francisco Lafarga, Albert Ribas y Mercedes Tricás, eds. La traducción: Metodología, historia, literatura: Ámbito hipanofrancés. Barcelona: PPU, 115-122.

Van Hoof, Henri (1991): Histoire de la traduction en Occident: France, Grande-Bretagne, Allemagne, Russie, Pays-Bas. Paris: Duculot.

VAN Hоoк, La Rue (1914): Greek rhetorical terminology in Puttenham's the Arte of English Poesie. Transactions and Proceedings of the American Philological Association. 45:111-128.

Venuti, Lawrence (1995): The Translator's Invisibility: A History of Translation. London/Nueva York: Routledge.

Vickers, Brian (1990): In Defence of Rhetoric. Oxford: Clarendon Press.

WARD, B.M. (1925): The authorship of the Arte of English Poesie: A Suggestion. Review of English Studies: A Quarterly Journal of English Literature and the English Language. 1(3):284-308. 